\title{
A Practical System Hardness Assurance Program
}

\author{
L. Henderson and L. Simpkins, MMC \\ A. Namenson, A. Campbell, and J. Ritter, NRL \\ and \\ E. Wolicki, Wolicki Associates, Inc.
}

\begin{abstract}
A hardness assurance program has been developed for space programs that is believed to be practical and cost effective. It includes estimates of a worst case natural and an enhanced radiation environment, addresses piece part selection and procurement, and provides methods for calculating parameter deratings for design hardening. The program emphasizes the crucial importance of examining the radiation response data with utmost care before any statistical inferences about performance and survivability are drawn from the data.
\end{abstract}

\section{Introduction}

The design hardening and hardness assurance programs required for space systems are often specified as contractual agreements between the system developer and the customer. But, although such contracts may refer to relevant government documents[1,2,3], existing documents usually do not adequately cover the problems that can arise for specific systems[4,5,6]. In addition the level of detail in specifications provided early in the program can vary widely from one customer to the next. At times, detailed specifications including environmental profiles, overtest tables and specified survival probabilities may be provided. At the other extreme, specifications on desired operational orbital characteristics and design life may be the only information available. In addition, the trend toward lower cost light weight spacecraft designs with ever increasing design life and performance requirements create increasing challenges for system developers. This paper is, therefore, presented because the recommendations and procedures which have been found useful for a generic space system program are expected to be useful also for particular systems.

Specifically, a hardness assurance program has been developed for a generic space system with a survivability specification which includes the natural space environment and possible enhancement due to a test such as Starfish. The program provides estimates of the worst case radiation environments, discusses criteria for selecting and procuring piece parts, and provides methods for calculating derated parameter values for circuit applications. The focus here is on system hardening from the point of view of total dose effects which along with Single Event Effects, represent generic concerns which must be addressed for all space systems. This program includes options which are helpful when various system tradeoffs need to be made and has been judged to be both cost effective and straightforward to use by system project officers.

\section{Hardening Process and Radiation Environment Specifications}

A diagram illustrating the total dose hardening process for an electronic space system is shown in Figure 1. A space system must be designed to operate with full capabilities, for a planned mission duration in a specified environment. For near earth orbits, the radiation environment includes the natural environment of trapped electrons and protons in the Van Allen belts around the earth, protons and other particles emitted from the sun during solar flares, and cosmic ray ions. The types and amounts of radiation that the system must survive depend on the orbital dynamics of the satellite, the solar sunspot cycle, the random occurrence of solar flares, and the mission duration. In addition, the system radiation specification can include the possibility of a test such as Starfish, in which case increased fluxes of particles in the Van Allen belts would occur. Models of all of these different radiation sources exist, such as the AP8 and AE8 environmental models available from the Space Science Data Center at NASA Goddard. However, they do not cover all of the dynamic behaviors of the radiation sources. It is, however, possible to estimate a worst-case radiation specification that a particular system will have to survive. As an example, with NASA models appropriate for a particular spacecraft orbit, the trapped proton environment is calculated for conditions of solar minimum, while the trapped electron environment is calculated for solar maximum, even though these two conditions cannot exist simultaneously. However, if system design life requirements or development schedules are such that operation is required throughout the solar cycle, then the increased fluxes of electrons and protons occurring during solar maximum and solar minimum must be considered. The solar flare intensity, again for a specified orbit, is based on previous worst-case measurements of solar flares. A separate model is used for expected enhancements due to a test such as Starfish. In system specifications, the radiation environments are described in terms of integral and/or differential particle spectra which describe as a function of energy, or linear energy transfer for cosmic rays and heavy solar particles, and 
Figure 1 - EXAMPLE OF TOTAL DOSE SYSTEM HARDENING APPROACH

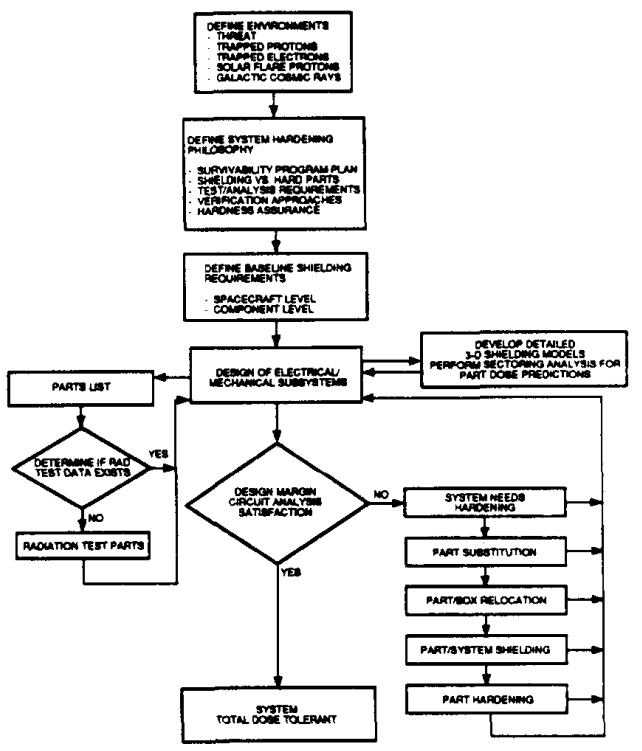

the omni-directional flux or fluence of particles which the spacecraft must endure. These specifications serve as the basis for derivation of lower level surface dose, subsystem and component/piece part total dose tolerance requirements and supporting analysis. The total estimated radiation level, integrated for the duration of the required mission lifetime, is termed the TR. It is the sum of two components, the natural radiation, NR, and the enhanced radiation, ER.

$$
\mathrm{TR}=\mathrm{NR}+\mathrm{ER}
$$

External to the spacecraft, the radiation environments are termed "free field" environments. Unless they are specifically identified as "free field" environments, it should be understood that TR, NR, and ER always refer to the radiation levels at a particular shielded location. How the shielding calculations are made is discussed in the paragraphs that follow.

Early in the system design, the spacecraft mechanical design and layout is in flux as various concepts and configuration alternatives are explored. However, performance requirements and cost considerations necessitate early identification of alternative device technologies and vendors. As a first step in the process, once the "free field" environments are defined, preliminary total dose shielding analyses may be performed to generate a system dose depth curve showing the dose absorbed as a function of uniform shield thickness. Based on these analyses, derived spacecraft skin and black box component minimum shielding requirements can be established with due consideration to available piece part technology, and volume and weight constraints. A spherical shield geometry is often assumed to establish uniform baseline shielding requirements and a corresponding uniform piece part hardness goal. The baseline minimum shielding requirements are then levied in the appropriate specifications and preference in piece part selection is given to device types available as a Radiation Hardness Assured (RHA) product from a qualified vendor. For such devices, vendors establish special processes, procedures and controls to minimize part-to-part variation and monitor their processes to ensure within specification post-irradiation performance at the specified total dose level.

A common occurrence in space programs is that a particular piece part with highly desirable characteristics is not adequately hard to the specified system radiation level and that no suitable RHA substitute part can be found. It is then up to system developer to establish the necessary piece part characterization and hardness assurance controls to ensure the survivability of the design and production hardware. The importance of establishing such controls cannot be overemphasized. In this situation, the only practical resort may be to make use of shielding to reduce the radiation level so the part will operate satisfactorily.

In the present example, sector shielding programs are used which can calculate the inherent shielding, at any location within the spacecraft, from isotropic "free field" radiation environments with specified characteristics. These detailed shielding calculations are then performed for the intended part location based on the three-dimensional mechanical shielding model of the system design. If the radiation level is still too high, a different location for the part is considered and a new calculation is made. The process is iterated (in effect a depth dose curve is developed for the system) until the best possible location, consistent with the mechanical and electrical design of the spacecraft, is found for the part. If the shielding at this location is still not adequate, then, as a last resort, the addition of local, small volume (minimum weight), shielding is considered. For LEO systems, the problem is often one of reducing the electron dose contribution and this is effectively accomplished using a high atomic number material such as lead (low atomic number shields are more effective per unit mass for electrons but high atomic number shields are more compact). If the part is used in several locations, the worstcase local shielded radiation level becomes the specified radiation level for that part type and is identified in the documentation for that part type. For that part type then, characterization test data is reviewed and analyzed to establish parameter deratings for two times the shielded radiation level.

For any part that requires shielding, configuration control must be strictly maintained throughout system production to ensure that the particular part type is never used in a less shielded location or that the local shielding, if that is required, is not inadvertently omitted. As a final step in the process, circuit analysis using the parameter deratings are performed to verify proper circuit performance of the hardened design. 


\section{System Survivability Control Plan}

A key consideration in the successful implementation of a system survivability program is the development of program documentation which facilitates understanding of the overall hardening process. In the generic system, a System Survivability Control Plan is established which describes the hardening process at the "Systems" level. It includes detailed characterization and lot acceptance test requirements, processes, and procedures; and establishes the roles and responsibilities of the various program organizations relative to survivability program implementation. The generic system has to meet functional and performance requirements over the design mission for ionizing radiation dose (total dose) and displacement damage effects from trapped protons, trapped electrons, and solar flare protons, and single event effects from trapped protons, solar flare protons, and galactic cosmic ray ions. Total dose and displacement damage effects result in permanent degradation in system performance so they are treated in terms of radiation design margins. Single event effects can include transient single event upsets (SEUs) in digital electronics and temporary or permanent damage from single event latchup (SEL), the latter occurring in bulk CMOS technologies. Analog bipolar and BICMOS technologies are also highly susceptible to SEL. The system must be able to tolerate SEUs or SELs if they occur.

The present plan emphasizes the use of overtests both for characterizing the radiation responses of the part types to be used in the system and for calculating derated parameter limits for circuit applications. In addition, overtests may be used for lot acceptance testing for those part types that require such tests.

\section{Radiation Response Data}

Radiation response characterization data should be obtained, for each manufacturer, for each part type, and, as a minimum, for 10 parts plus a control from each of three date codes separated successively in time by no less than 6 weeks. This sampling is intended to reveal variations between manufacturers and between lots from each manufacturer over time. To ensure that the part type will not be subject to abrupt failure at a level just above its intended use, step stress data should be obtained either up to failure or to 10 times the expected worst case shielded part level, whichever is greater. The step stress data should include the three levels: $2 \mathrm{NR}, 5 \mathrm{NR}$, and $10 \mathrm{NR}$.

\section{Examination of Radiation Response Data}

It is crucial to scrutinize data with utmost care. This caution is considered essential in light of the problems that have been found on many occasions in radiation response data that had been assumed to be satisfactory. Indeed, accumulated experience in the radiation effects field would indicate that completely satisfactory radiation response data is generally the exception and not the rule. It is important to note in this connection that problems found with measured data may not be due to measurement errors or poor technique but, rather, to the high sensitivity of device radiation response to slight changes in device geometry and processing parameters; so much so, that, in some technologies, radiation response measurements can be used to gauge whether processing is under control. It should be noted that statistical inferences about system or piece part performance and survivability may be grossly in error if a careful examination of the data does not show it to be satisfactory.

An approach to data examination and some of the most common problems that occur are shown in Figure 2. The possibility of an abrupt failure just above the system application level has already been mentioned. In addition, the data must be examined to detect measurements that may be in error and to detect outlier data points. For any suspect points, attempts should be made to discover the reason for the value found. A measurement that is in error must be eliminated from the data before further analysis is performed. An outlier data point however that is not an erroneous measurement cannot be removed from the data and can constitute a serious problem. The number of outliers that may be present in the part populations will have a direct effect on the probability and confidence levels with which statistical estimates of device performance can be made, and will also impact lot acceptance tests. The problem is worse when only small sample sizes are available for testing because the probability of detecting an outlier will be roughly proportional to the number of devices tested. A "rule of thumb" for the lower limit on the probability that outliers are present in the population is to equate it to the number of outliers found in the measurements plus one divided by the total number of parts tested.

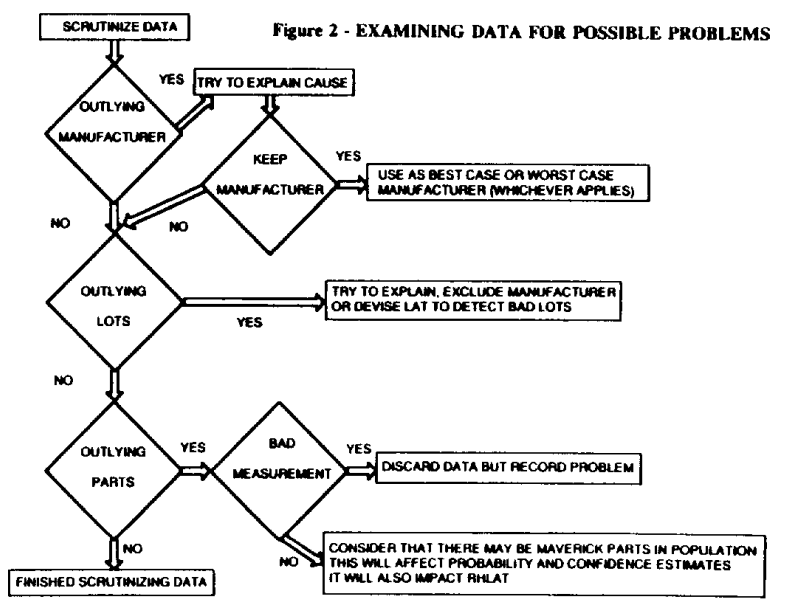


It may also be true that all the measurements are satisfactory, but the parts from one or more particular manufacturers will show a degraded response that is well outside the ranges for other manufacturers. In such cases it may be advisable to approve only the most qualified manufacturers. In common with most other hardness assurance programs, the present program requires that the system be produced only from parts and manufacturers contained in the Approved Parts List, or APL.

A generally useful and practical technique for examining the data is to begin by constructing plots of the relevant data on probability paper. Such plots are remarkably effective in identifying worrisome features in the data and can be used to discover the various problems mentioned above. Thus, distributions which are not normal (or lognormal), outliers, bad measurement, unacceptable variations from lot-to-lot, within a lot, and between manufacturers, all are conditions in the data that have been found by means of probability plots. These plots, which can be obtained quite quickly, often give results that are as informative as much more elaborate procedures. Figure 3 shows a probability plot with confidence lines of measurements of Iebo after a total dose exposure of $100 \mathrm{krad}(\mathrm{Si})$ for one particular transistor type from three manufacturers, 4 or 5 individual devices per manufacturer. Also shown is the manufacturers guaranteed limit for this parameter, $200 \mathrm{nA}$. It can be seen from the figure that manufacturer $A$ (ovals) shows a large variation in Iebo values and manufacturer $\mathrm{C}$ (diamonds) shows values outside the acceptable range (10\% to $90 \%)$. Thus only manufacturer B (squares) should qualify.

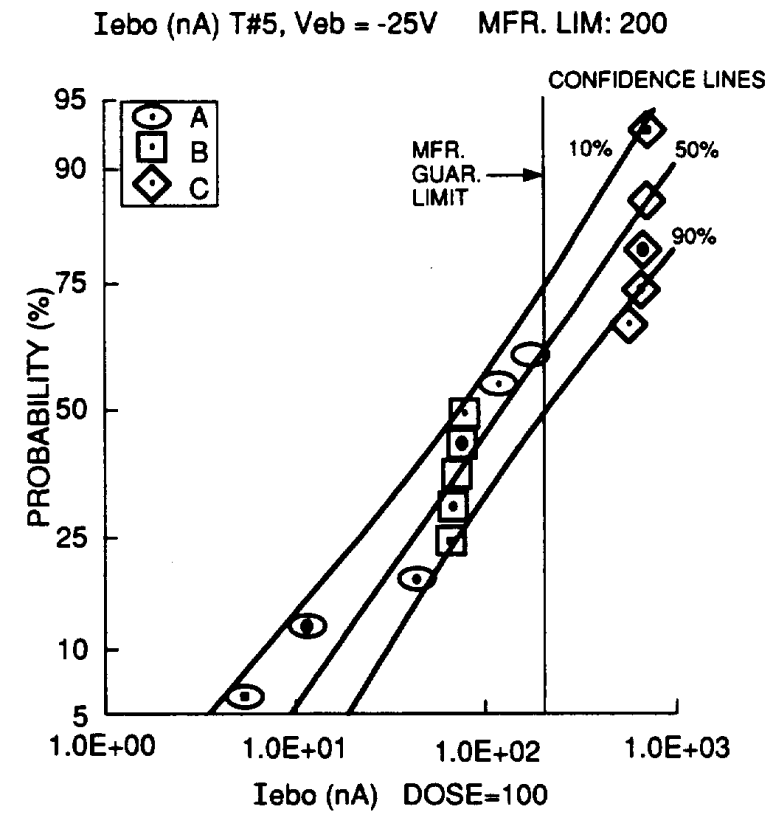

FIGURE 3: LOGNORMAL PROBABILITY PLOT FOR 3 MANUFACTURERS AND SAME DEVICE TYPE ONLY MANUFACTURER B QUALIFIES.
Figure 4 shows a probability plot for measurements of transistor gain (100/transistor gain or $100 / \mathrm{hFE})$ shift (DEL or delta) for the same device type from manufacturer $\mathrm{C}$ for three date codes after $50 \mathrm{krad}(\mathrm{Si})$ exposure which clearly reveals two outlier measurements (that turned out to be bad measurements). Figure 5 shows that the data fits a lognormal distribution quite well when the two erroneous points are eliminated.
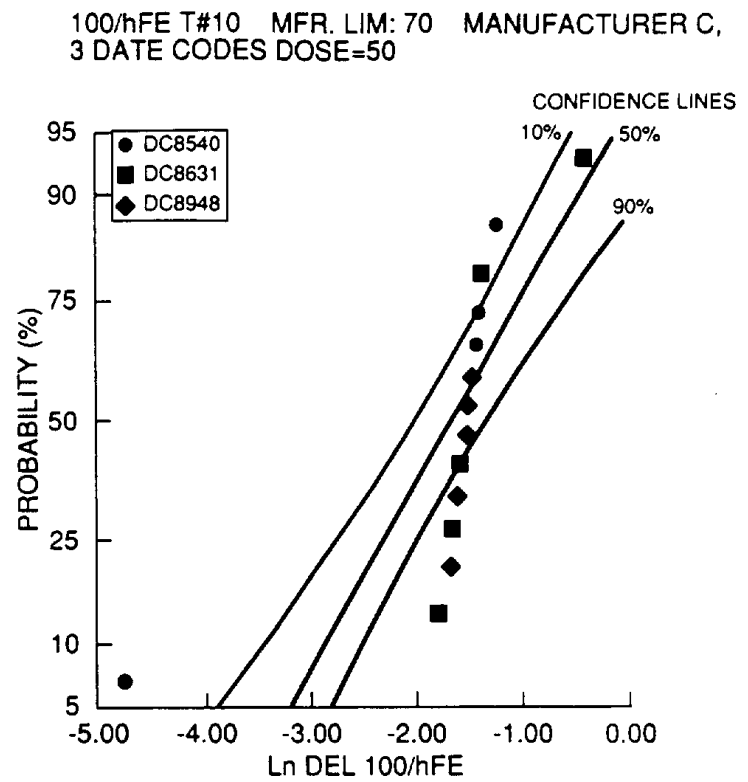

FIGURE 4: LOGNORMAL PLOT FOR 3 DATE CODES FROM A SINGLE MANUFACTURER. THERE ARE 2 OUTLIERS BOTH DETERMINED TO BE BAD MEASUREMENTS.

\section{Piece Part Selection, Design Derating and Hardness Assurance Processes}

After any unacceptable manufacturers and erroneous points have been removed, and a careful examination has shown the characterization data to be satisfactory, such data will be used for supporting parts selection, lot acceptance tests, and calculating design deratings or derated parameter limits.

In the generic system, initial baseline worst case piece part ionizing dose requirements for preliminary piece part selection and screening are derived from an analysis of the system depth-dose curve and consideration of available piece part technology and system weight constraints.

Total dose radiation hardening is accomplished through a combination of piece part selection and test, radiation shielding analysis and design, and piece part parameter derating. The dose failure level is that level at which the part causes unacceptable circuit performance. Part selection 
100/hFE T\#10 MFR. LIM: 70 MANUFACTURER C, 3 DIFF. DATE CODES -- REMOVE 2 OUTLIERS

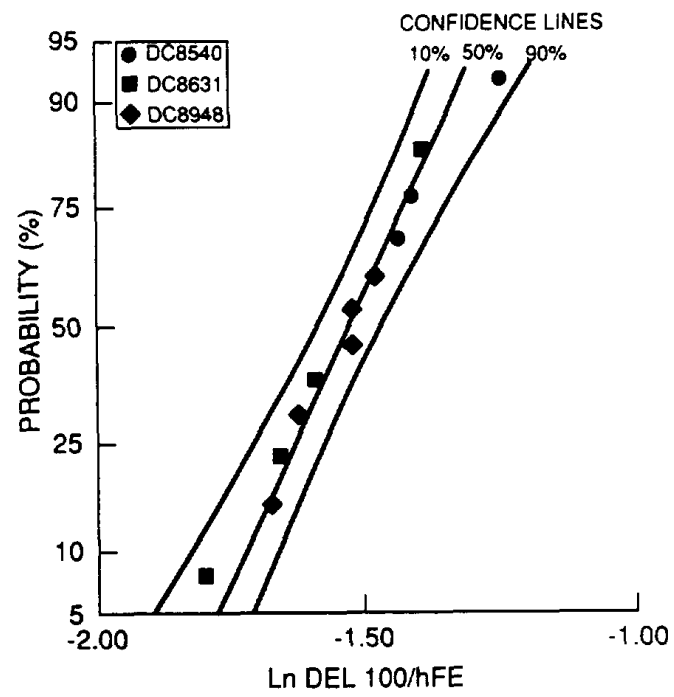

FIGURE 5: SAME AS FIGURE 4 BUT OUTLIERS REMOVED

decisions are made on the basis of design margins which are defined as follows:

The Natural Radiation Design Margin, NADM is:

$$
\text { NADM }=\text { Dose Failure Level } / \text { NR, }
$$

And the Total Radiation Design Margin, TDM is:

TDM = Dose Failure Level $/$ TR.

A policy used in the generic program is that nonradiation hardened devices are unacceptable for a particular device location if NADM is $<2$ or if TDM $<1$, whichever corresponding fluence is greater.

This is a procedure which has not been adopted in any previous program. Separating the design margin into the natural environment component and the enhanced environment component allows a program to decide what confidence level to meet each environment. A program could thus decide to meet the natural environment with a high degree of confidence, but to meet the enhanced environment with a much lower degree of confidence, since it is much less likely to occur.

Table 1, below, summarizes criteria for lot acceptance testing that are believed to be reasonable and cost-effective.
Table 1: Total dose characterization and hardness assurance requirements. Characterization test data on 3 lots, based on date codes spaced at least 6 weeks apart (10 parts per lot), for each manufacturer.

\begin{tabular}{|c|c|c|}
\hline $\begin{array}{l}\text { CATEGORY } \\
\text { REOUIREMENTS }\end{array}$ & NADM & $\begin{array}{llll}T & E & S & T\end{array}$ \\
\hline HCC-1 & $\geq 2<5$ & $\begin{array}{l}\text { w a f e } r \text { lot } \\
\text { a c e pta n e } \\
\text { testing }\end{array}$ \\
\hline HCC-2 & $\geq 5<10$ & $\begin{array}{l}\text { date code lot } \\
\text { acceptance testing }\end{array}$ \\
\hline HNC & $\geq 10$ & $\begin{array}{l}\text { all part types } \\
\mathrm{r} \text { e } \mathrm{q} \text { u } \mathrm{i} \text { e } \\
\text { characterization } \\
\text { testing. }\end{array}$ \\
\hline
\end{tabular}

HCC-1 (hardness critical category 1). Parts which fall into this category have low design margins such that marginal lots might result in unacceptable jeopardy to the system hardness. HCC-1 piece parts, thus, require wafer lot hardness assurance testing on each part type and manufacturer used in the flight hardware to reduce the chances that a marginal lot will be purchased.

HCC-2: These parts have reasonable design margins and as such represent a lower concern than HCC-1 parts, but are subject to periodic total dose lot acceptance testing, such as, for example, at the date code level, on each part type and manufacturer used in the flight hardware.

HNC: (hardness non critical) parts have large design margins such that the risk of compromising system survivability under natural radiation conditions, because of performance variations from lot-to-lot, is deemed low. Characterization test data are required initially for the part types and manufacturers identified for use in the production hardware. Additional testing may be required at the discretion of the hardness assurance working group or the parts, materials and processes control board if the data are judged to be out of date or if it is suspected that the manufacturer has made changes in his processes which could impact the part's total dose response.

HCC1-S. In the generic program, even if the design margin qualifies a part type as $\mathrm{HCC} 2$ or $\mathrm{HNC}$, because parts may be purchased only from approved manufacturers and controls are needed to ensure that this requirement is satisfied, all parts may be considered to be HCC1-S, a control category defined in MIL HDBK 280[2].

In a traditional Hardness Assurance program as outlined in MIL-HDBKS-279 and 280[1,2] (DNA Reports 5909 and 5910), a device would not be considered Hardness Non Critical (HNC) with design margins less than 100 . However, in many actual systems, total dose design margins on the order of 100 are hard to obtain due to the severity of the environments and the need to minimize shield weight. Thus, in the generic program, lot acceptance testing may be waived if the natural design margin is $\mathbf{1 0}$ or greater and the part is 
purchased only from an approved manufacturer. In addition, the waiver of lot acceptance tests may be subject to the part categorization criterion, PCC, as described in DNA Report 5910. This criterion depends on the variability of the radiation failure doses (PCC increasing in value as the variability increases) and requires lot acceptance tests if the design margin does not exceed the PCC. The risk introduced in the generic system plan by the lower required design margins is, therefore, offset somewhat by the limitation of qualification approval to certain established manufacturers and by the application of the PCC to identify devices types requiring radiation lot acceptance testing. This procedure minimizes additional risk and can result in a substantial cost saving.

The generic plan requires that all parts used for system production be taken from an approved parts list, APL, and that the placement of parts into production stocks be based either on lot acceptance tests or radiation response data. Preference in design is given to devices procured as a radiation hardened product to a specified dose level to minimize potential hardness assurance costs and risk.

Flow diagrams illustrating the piece part test data review and analysis subprocess are shown in Figures $6 \& 7$. The term "cherry picking" in figure 6 refers to the use of electrical tests for selecting parts with some parameter better than the manufacturers guaranteed limit. Thus, for example, if the manufacturers guaranteed limit on gain is 200 , the parts may be tested so that only parts with gains greater than 250 are selected ("cherry picked").

\section{Figure 6 - LOT ACCEPTANCE CRITERIA}

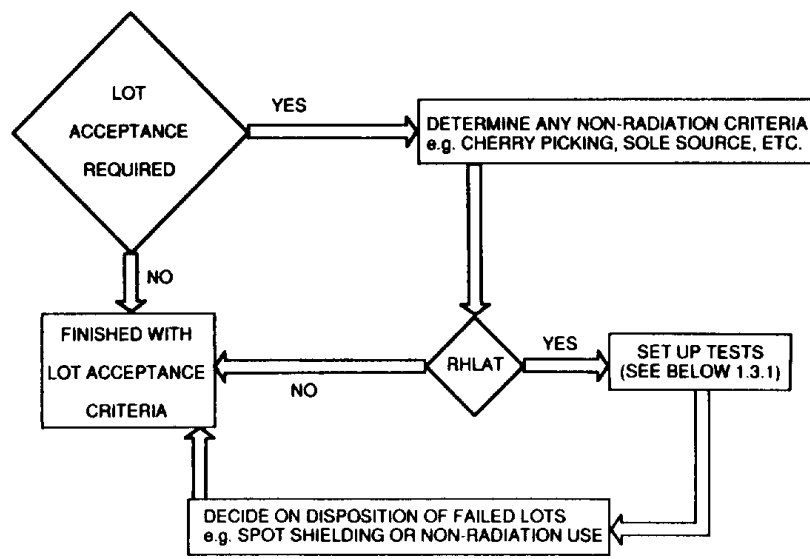

Thus, the plan requires that if a radiation hardened (TDM > 1) class $\mathrm{S}$ device is available, it shall be used. For non-radiation hardened devices, whenever possible, derated parameter limits for design are established at 10NR or 1TR, whichever fluence is greater, based on analysis of piece part characterization test data representative of the flight device and manufacturer. This approach was selected in order to gain some insight into potential lot-to-lot variability over time and into the possibility of abrupt failure.
Figure 7 - FLOW DIAGRAM FOR LOT ACCEPTANCE TESTS 1.3.1 SET UP RHLAT TESTS COST-EFFECTIVE TRADE OFFS BETWEEN RISK \& EXPENSE

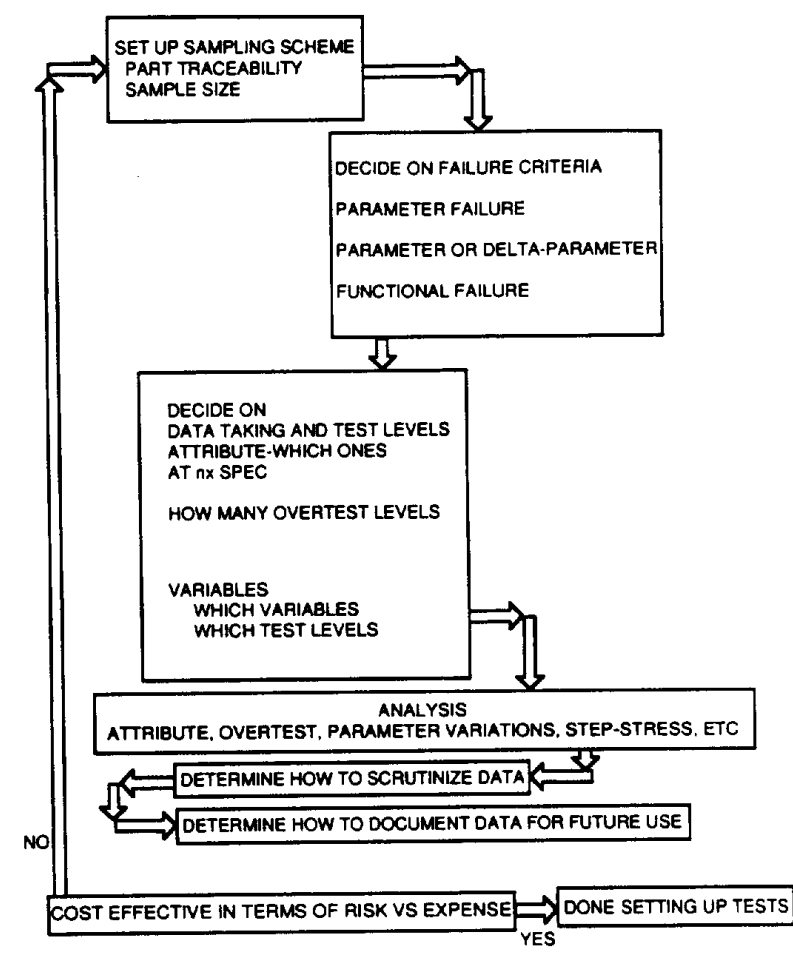

\section{End Point Limits, Lot Acceptance Tests, and Derated Parameter Limits}

A critical feature of the plan is the recognition that system survivability must be based on actual radiation response data for each part type to be used which is of adequate quality and includes information not only about failure levels or parameter degradations but also about the several kinds of response variabilities that will be found in practice[7].

The radiation response characterization data will be used for three main purposes:

a) To set the end point limit, EPL, against which future lot acceptance tests will be performed. It should be understood, however, that engineering judgement needs to be applied here because a trade off is necessary to balance the need for multiple sources to meet program build schedule requirements (i.e. flexibility) against the need to allow circuit designers to take advantage of using only the parts or manufacturers which have shown high levels of radiation hardness and small variabilities. The basic purpose of the EPL is to eliminate the few lots that are clearly unacceptable but, otherwise, to allow most lots from approved manufacturers to pass.

b) To decide whether a part type needs to undergo lot 
acceptance tests and

c) To obtain the derated parameter limit, DL, which the circuit designer can use for his hardened design. The derated parameter limit is based on the radiation response data and is a statistical limit such that with confidence, $C$ (which is often 90 percent), a fraction $P$ (which is typically 0.95 or greater) of the parts will not be expected to degrade beyond the limit after the specified radiation dose. The derated parameter limit is often set at the same numerical value as the EPL. The two numbers actually have different purposes, however, and this difference should be understood. Thus, for example, MIL STD procurement specifications, so called "slash sheets," include an EPL for lot acceptance tests, but that EPL cannot be used as a basis for circuit design with the same confidence as the DL which is based on current radiation response data, even though, numerically, the values of the DL and the "slash sheet" EPL may be the same.

\section{End Point Limit}

The end point limit, EPL is usually set so that most of the lots to be tested may be expected to pass. A necessary caution is to observe, that if EPL is set so that most lots are failing, then there may be a significant probability that even those few lots that are passing are only statistical anomalies and would not pass if they were subjected to more extensive tests, e.g. tests on larger sample sizes. Once a value is set for EPL, that same value is used for all approved manufacturers. Knowledge about the passed lots improves as lot acceptance data accumulates.

Various options may be used for obtaining EPL. Usually, EPL is obtained from a statistical calculation based on the characterization data and adjusted so that, with confidence $C$, a fraction $P$, of the parts to be tested will pass the EPL value after irradiation. If lot to lot variations are large, a multi lot analysis[8] may be performed to obtain an EPL such that the desired fraction of the tested lots will pass.

Other options include making the statistical EPL calculation (and performing the corresponding lot acceptance tests) at an XNR radiation overstress level for an application at the NR level. Performing the lot acceptance tests at an overstress level ensures that the accepted lot will not suffer an abrupt failure at the NR radiation level and can require smaller sample sizes. The use of data from just the worst case approved manufacturer is still another possibility.

For a normally distributed parameter that increases with increasing radiation (please note that such behavior will be asumed in the rest of this paper and that a minus sign should be used if the parameter value decreases with radiation), EPL(XNR) can be obtained from:

$$
\begin{array}{r}
\operatorname{EPL}(\mathrm{XNR})=\operatorname{MEAN}(\mathrm{PAR}, \mathrm{XNR})+\operatorname{KTL}(\mathrm{n}, \mathrm{C}, \mathrm{P}) \\
\operatorname{STDEV}(\mathrm{PAR}, \mathrm{XNR})
\end{array}
$$

where:

MEAN(PAR,XNR) is the arithmetic mean value of the measured post-rad values at $X$ times the natural radiation level, NR,

$\mathrm{KTL}(\mathrm{n}, \mathrm{C}, \mathbf{P})$ is the one sided tolerance limit factor (which may be found in standard statistical tables) for sample size, $n$, confidence $C$, and part survival probability, $P$, and

STDEV(PAR,XNR) is the standard deviation of these same values.

For a parameter that is lognormally distributed, LN(EPL) is first calculated by the equation:

$$
\begin{array}{r}
\mathrm{LN}(\mathrm{EPL}, \mathrm{XNR})=\operatorname{MEANLN}(\mathrm{PAR}, \mathrm{XNR})+\mathrm{KTL}(\mathrm{n}, \mathrm{C}, \mathbf{P}) * \\
\text { STDEV }(\mathrm{LN}(\mathrm{PAR}, \mathrm{XNR}))
\end{array}
$$

where:

MEANLN(PAR,XNR) is the mean logarithm of the measured post-rad parameter logarithms at $\mathrm{XNR}$, and $\mathrm{KTL}(\mathrm{n}, \mathrm{C}, \mathrm{P})$ is the same as in the previous equation.

EPL(XNR) is then found from:

$$
\operatorname{EPL}(\mathrm{XNR})=\exp (\mathrm{LN}(\mathrm{EPL}, \mathrm{XNR}))
$$

\section{Lot Acceptance Test}

The characterization data for each manufacturer should be examined to determine whether lot acceptance tests need to be performed for that manufacturer. To minimize the number of part types that will need to be subjected to lot acceptance tests, the PCC method discussed in DNA Report 5910 , may be used. This method uses design margins and assumes that the failure fluences are lognormally distributed. Lot acceptance tests may be waived for a part type (or even a particular manufacturer) if:

$$
\mathrm{DM} \geq \mathrm{PCC}=\exp (\operatorname{STDEV}(\mathrm{LN}(\text { Failure Fluences }))
$$

where DM is either of the design margins, NADM or TDM, defined earlier,

PCC is the part categorization criterion, and STDEV(LN(Failure Fluences) is the standard deviation in the natural logarithms of the failure fluences measured for a part type (or a particular manufacturer).

Preferably, lot acceptance tests should be performed as a variables test in which a statistical limit value for the lot:

$$
\begin{array}{r}
\operatorname{SLV}(\text { lot,XNR) }=\operatorname{mean}(\operatorname{PAR}(\text { lot }), \mathrm{XNR}) \\
\text { KTL(n,C,P)*STDEV(PAR(lot),XNR) }
\end{array}
$$

is compared to EPL(XNR); the lot is passed if SLV(lot,XNR) < EPL(XNR). However, lot acceptance tests may also be performed by an attribute test (such as the MIL STD Lot Tolerance Percent Defective (LTPD) test) in which case the post-irradiation parameter value of each part is compared to EPL. 


$$
\operatorname{DDL}(\mathrm{XNR})=\operatorname{MEAN}(\mathrm{DELTA}, \mathrm{XNR})+\mathrm{KTL}(\mathrm{n}, \mathrm{C}, \mathrm{P}) *
$$$$
\text { STDEV(DELTA,XNR) }
$$

As has been mentioned, the derated parameter limit, $\mathrm{DL}$, is the parameter value which the circuit designer will use for his design. He will thus assume that, for any particular part type in its worst case locations, the derated parameter will not degrade beyond the estimated limit. One conservative approach to calculating the $\mathrm{DL}$ is to use the pre- and post-irradiation changes in a parameter value, the so called deltas, together with the manufacturer's guaranteed limit.

Thus:

$$
\begin{array}{r}
\text { DMDL }(\mathrm{XNR})=\mathrm{MGL}+\mathrm{MEAN}(\text { DELTA, XNR) } \\
\text { KTL(n,C,P)*STDEV(DELTAS, XNR) }
\end{array}
$$

where:

DMDL(XNR) is the notation used to identify that this design margin is calculated for delta parameters and the manufacturers guaranteed limit at radiation level XNR, MGL is the manufacturer's guaranteed limit, MEAN(DELTA, XNR) is the mean parameter change between its pre rad value and its value after irradiation to XNR (note: the deltas must be obtained for each device separately),

$X$ is the overstress level and should be $\geq 2$, and

STDEV(DELTAS, XNR) is the standard deviation in the delta parameters.

It should be noted that, here again engineering judgement must be used to ensure that an unduly restrictive derated parameter limit is not imposed on the circuit designer. Too restrictive a DL may result in increased circuit complexity, higher power dissipation, and/or degraded hardware performance. In most cases the radiation derating factor is but one of many derating factors (e.g. end of life or aging, stress, temperature, etc.) imposed upon the circuit designer. It may even result in a weight penalty through the need for local or spot shielding which a less restrictive derated limit might not require. In practice, and with the aforementioned caution exercised about avoiding unduly restrictive derated parameter limits, the derated parameter limit DL is often made the same as the value calculated for the end point limit EPL.

A second important feature of the present plan is the use of derated parameter limits based on data measured at radiation overtest levels for hardening circuit designs. Thus, if a derated parameter value is calculated from data measured at $\mathrm{X}$ times the estimated radiation level for the system, the survivability at 1 times that level will be greatly increased. In practice and depending on the variability measured, even at a 5 times overtest, the part survival probability may increase so substantially that the major uncertainty in the part survival probability may then be due to the probability that maverick parts, i.e. parts that will fail at anomalously low radiation levels, will be present in the production stock of parts. A derated parameter limit, DDL is calculated by means of the formula: where:

DDL(XNR) is the notation used to signify that delta parameters are being used,

MEAN(DELTA,XNR) is the mean change in the parameter pre- and post-irradiation at $\mathrm{X}$ times the specified stress level NR,

$\mathrm{KTL}(\mathbf{n}, \mathbf{C}, \mathbf{P})$ is the one sided tolerance factor as before. C often equals 0.90 and $P$ is often 0.99 or 0.999 ,

STDEV(DELTA,XNR) is the standard deviation of the deltas for the sample.

This calculation should be made in the probability distribution that is best for the data in question; in many cases, for example, the lognormal distribution is the preferred one.

Caution: If delta parameters are used, the data must be examined to make sure that negative deltas are not appearing in the data. In the event that some device parameter actually improves with radiation (before finally degrading when the radiation level gets high enough), the parameter values themselves should be used. A check should also be made on the probability distribution which applies.

The derated parameter limit at XNR, DPL(XNR), may also be established based on the post irradiation parameter values instead of deltas as follows (this option may be useful if negative deltas are occurring):

$$
\begin{gathered}
\operatorname{DPL}(\mathrm{XNR})=\operatorname{MEAN}(\mathrm{PAR}, \mathrm{XNR})+\mathrm{KTL}(\mathrm{n}, \mathrm{C}, \mathrm{P}) * \\
\text { STDEV }(\mathrm{PAR}, \mathrm{XNR})
\end{gathered}
$$

where:

MEAN(PAR,XNR) is the mean post-irradiation parameter value at $X N R$, and

STDEV(PAR,XNR) is the standard deviation in the postirradiation parameter values at XNR.

For any of the above three derated parameter limits, if the calculation is made at an overstress level, i.e. $X \geq 2$, then a significant increase in the part survival probability is produced by applying that DPL at one times the specified stress level. In addition, the overstress testing ensures that abrupt failure will not occur at 1NR. If there is no data at 1NR, the standard deviation found at $\mathrm{XNR}$ and the value of the part survival probability, $\mathrm{P}$, used in the KTL factor at $\mathrm{XNR}$, could be used in the overstress formula[9] to estimate the increased survival probability at $1 \mathrm{NR}$. If data at $1 \mathrm{NR}$ is available, the derated parameter limit formulas given above can be used with any desired values for the confidence $C$, and part survival probability $P$. Using the derated parameter limit DPL(XNR) at $1 \mathrm{NR}$ will then simply correspond to using an increased value for $P$ so that DPL(1NR) equals DPL(XNR). Thus, if:

$$
\begin{gathered}
\mathrm{DPL}(\mathrm{XNR})=\operatorname{MEAN}(\mathrm{PAR}, 1 \mathrm{NR}) \pm \mathrm{KTL}(\mathrm{n}, \mathrm{C}, \mathrm{P}(\mathrm{new})) \\
\text { STDEV}(\mathrm{PAR}, 1 \mathrm{NR})
\end{gathered}
$$


then $P$ (new) will be larger than the $P$ used at XNR.

The following discussion, based on Iebo data, illustrates how $\mathrm{P}($ new) increases. For the $\mathrm{KTL}(\mathrm{n}, \mathrm{C}, \mathrm{P})$ at $50 \mathrm{Krads}$, assume sample size $n=5, C=0.9$, and $P=0.95$, then KTL $=3.400$. Let $\mathrm{NR}=10 \mathrm{Krads}, \mathrm{XNR}=50 \mathrm{Krads}$, the MEAN(Iebo, $50 \mathrm{Krads})=33.22 \mathrm{nA}$, the STDEV(Iebo,50 Krads $)=5.876 \mathrm{nA}$, MEAN(Iebo,10 Krads $)=13.88 \mathrm{nA}$, and STDEV(Iebo,10 Krads) $=1.580 \mathrm{nA}$. Then, the DPL at 50 Krads, is:

$$
\operatorname{DPL}(50 \mathrm{Krads})=33.22+3.400 * 5.876=53.198
$$

And this DPL is to be used at 10 Krads. Thus:

$$
53.198=13.88+\operatorname{KTL}(5,0.9, \mathrm{P}(\text { new })) * 1.580
$$

Here the new KTL is 24.88 and if $n=5$ and $C=0.9, P$ must be $=0.999$. Thus, in this case, the part survivability $\mathbf{P}$, increased from 0.95 at $50 \mathrm{Krads}$ to 0.999 at $10 \mathrm{Krads}$.

\section{Single Event Effect Assessment and Hardening}

In addition to potential total dose degradation or failure Single Event Effects (SEE) induced in system electronics represent a second major area of concern for a generic spacecraft system. Single Event Effects such as SEU and SEL may result from the interaction of a single high energy nuclear particle with a sensitive region internal to the device. Depending on the devices used and the circuit/system design, system effects can range from minor ones such as an occasional correctable SEU to catastrophic failures. Numerous space systems designers have learned the hard way that attention to SEE must occur early in the design process if anomalous on-orbit operations or expensive redesign are to be avoided. Unfortunately, the relative lack of SEE test data and the fact that not all SEU's may have a noticeable impact on system operations complicates the matter. Nevertheless, as device feature size decreases and speed increases, devices are becoming more vulnerable to SEE and these effects continue to be a growing challenge for system developers.

For the generic system it is the natural proton and heavy ion environments which are of concern. The Single Event Effects assessment and hardening process begins with definition of the galactic cosmic ray, solar flare proton, and geomagnetically trapped proton environments for the orbit of interest which are then included in the appropriate system specifications. Next, baseline requirements and criteria for piece part selection are derived and implemented in the piece part selection process. For the generic system, devices susceptible to destructive SEL may not be used. All bulk CMOS technology devices are required to be evaluated for SEL susceptibility.

Preference is given to devices possessing a high degree of SEU tolerance. For the generic system, to verify that system performance and effectiveness are not degraded, single event vulnerability assessments are conducted and SEU and/or nondestructive SEL rates are calculated by using the CREME or Space Radiation computer codes for all device applications where the heavy ion threshold linearenergy-transfer (LET) is estimated to be less than 40 $\mathrm{MeV} / \mathrm{mg} / \mathrm{cm}^{2}$. This value is somewhat arbitrary but has been chosen because it is a convenient value for accelerator testing and because it represents a high value relative to the cosmic ray spectra even at geosynchronous orbit. Thus, in most cases, it represents an overtest value. When test results are combined with an estimated flux of cosmic ray ions with LETs greater than $40 \mathrm{MeV} / \mathrm{mg} / \mathrm{cm}^{2}$, it is straightforward to estimate the probability of an SEE event over a mission lifetime.

Devices with heavy ion threshold LET values of less than $15 \mathrm{MeV} / \mathrm{mg} / \mathrm{cm}^{2}$ are assumed to be potentially susceptible to proton induced SEU/SEL and are subject to proton testing if adequate proton test data is not available. Worst case peak proton upset rates during solar flare conditions or passes through the South Atlantic Anomaly (SAA) region, are assumed to cause 15 times the orbital average upset rate obtained from Bendel calculations[10] using the AP8 solar minimum model. The factor 15 comes from calculations of the ratio of of average proton flux over many orbits to the peak flux in the SAA for test orbits. When piece part SEE characterization testing is required, heavy ion testing is performed on a minimum of 3 devices representative of the flight manufacturer using ATSM F1172 as a guide.

Piece part selection and control are maintained through adherence to an approved piece parts list which identifies device types and manufacturers which have been evaluated in accordance with the plan and approved for use. The list identifies devices which represent a potential SEE risk and provides piece part parameter deratings for design.

In the event that SEU/SEL Vulnerability assessments result in unacceptably high predicted upset rates or probabilities, SEU/SEL countermeasures such as current limiting, error correction and detection, Majority Voting, memory refresh etc. are evaluated and implemented to ensure acceptable system performance.

\section{Conclusion}

A practical systems level survivability program approach for a generic space system has been developed which describes the process from the early program phases through piece part procurement and test. The program suggests reasonably conservative environmental definition strategies and preliminary total dose radiation shielding and dose tolerance requirements definition approaches which may then be modified as knowledge of the system mechanical shielding design matures. This serves to minimize shield weight impacts, while still allowing controlled usage of high performance, but radiation soft, technology devices when suitable RHA devices are unavailable. The program is flexible, placing preference on bonafide RHA device 
technology when available or on devices with reasonably high deign margins, and establishing increasing levels of control as radiation design margins decrease. Test data representative of contemporary devices confirms that many of the old familiar concerns regarding device-to device, manufacturer-to-manufacturer and lot-to-lot variability over time are alive and well. In light of this, the proposed program provides the necessary analytical methodologies to assess and evaluate the data quickly with respect to the numerous real life challenges encountered in Hardness Assurance programs. The program recognizes that new technologies such as GaAs digital devices which offer speed and power advantages in addition to high total dose tolerance nevertheless represent an increasing challenge for space system designers due to increased SEU susceptibility. Although developed for a generic LEO spacecraft application the program illustrates the generic hardening process and addresses the two generic radiation environmental effects which are common to all spacecraft applications.

This paper introduces a procedure which has not been used previously. The design margin is separated into a natural environment component and an enhanced environment component. This approach enables a program to assign different confidence levels to meeting each environment. It is certain that the natural environment will be encountered and hence the levels it produces must be satisfied to a high degree of confidence. Changing political conditions may introduce wide swings in the estimate of the need for also meeting an enhanced environment. This procedure allows for the probability of meeting each separate environment to be varied depending on the program's perceived requirements.

The paper also introduces different part acceptance criteria based on the natural environment design margin. Different environments require different test levels and parts from other programs may have been tested at levels which are an overtest for the program under consideration. This paper used derated parameter limits based on overtest levels to increase the survivability probability at the expected dose level.

\section{References}

1. W. E. Price, K. E. Martin, and M. K. Gauthier, "TotalDose Hardness Assurance Guidelines for Semiconductor Devices," DNA 5909F, 1 February 1982 and MIL HDBK 279 2. A. Namenson, E. Wolicki, R. Berger, H. Eisen, J. Ferry, G. Messenger, R. Scace, and H. Schafft, "Piece Part Neutron Hardness Assurance Guidelines for Semiconductor Devices, DNA5910F, 6 October 1981 and MIL HDBK 280

3. M. Rose, J. Harrity, N. Rudie, K. Rasmussen, and J. Halpin, "Design Guidelines for Transient Effects on Tactical Army Systems," HDL-CR-81-015-1, July 1981

4. G. C. Messenger and M. S. Ash, "The Effects of Radiation on Electronic Systems," Van Nostrand Reinhold Co., New York, 1986, Chapters 11, 12, and 13
5. F. N. Coppage, "System Development Hardness Assurance Guideline Document: Nuclear and Space Radiation Effects Aspects," to be published as MIL HDBK 817

6. J. Ferry, H. Eisen, and G. Messenger, "Gamma Dose and Neutron Hardness Assurance Guidelines," to be published as MIL HDBK 814

7. E. A. Wolicki A. Namenson and A. Carlan, "Guidelines for Developing Specifications for Radiation Hardness Assured Devices," to be published as MIL HDBK 816

8. I. Arimura and A. I. Namenson, "Hardness Assurance Statistical Methodology for Semiconductor Devices,"IEEE Trans. Nucl. Sci. NS-30, 4322 (December 1983)

9. A. I. Namenson. "Hardness Assurance and Overtesting," IEEE Trans. Nucl. Sci. NS-29, 1821 (December 1982)

10. W.L. Bendel and E.L. Petersen, "Proton Upsets in Orbit," IEEE Trans. Nucl. Sci. NS-30, 4481 (December 1983) 